\title{
Painter Classification Using Genetic Algorithms
}

\author{
Erez Levy \\ Dept. of Computer Science \\ Bar-Ilan University \\ Ramat-Gan 52900, Israel \\ levier@cs.biu.ac.il
}

\author{
Omid David \\ Dept. of Computer Science \\ Bar-Ilan University \\ Ramat-Gan 52900, Israel \\ mail@omiddavid.com
}

\author{
Nathan S. Netanyahu ${ }^{1}$ \\ Dept. of Computer Science \\ Bar-Ilan University \\ Ramat-Gan 52900, Israel \\ nathan@cs.biu.ac.il
}

\begin{abstract}
This paper describes the problem of painter classification. We propose solving the problem by using genetic algorithms, which yields very promising results. The proposed methodology combines dimensionality reduction (via image preprocessing) and evolutionary computation techniques, by representing preprocessed data as a chromosome for a genetic algorithm (GA). The preprocessing of our scheme incorporates a diverse set of complex features (e.g., fractal dimension, Fourier spectra coefficients, and texture). The training phase of the GA employs a weighted nearest neighbor $(\mathrm{NN})$ algorithm. We provide initial promising results for the 2- and 3-class cases, which offer significant improvement in comparison to a standard nearest neighbor classifier.
\end{abstract}

\section{INTRODUCTION}

Art forgery, which dates back more than two thousand years, has played a key role in the development of painting authentication. This task has been usually performed manually by art experts who have dedicated their lives to this profession. Their expertise amounted to using various characteristics other than what the human eye can see, including chemical analysis, spectrometry, and infra-red or X-ray imaging. The infamous Vermeer forgery [25] attests, perhaps, most vividly to the challenges presented by painting authentication. Han van Meegeren used historical canvasses and managed to deceive art experts into believing that his painting was an authentic Vermeer. Only after being charged with treason and sentenced to death for selling another (forged) Vermeer, did he confess and was forced to create another painting to prove himself innocent of treason. A more recent case of painting authenticity uncertainty is of the Pollock paintings found a decade ago in a storage locker in Wainscott, NY. The authenticity of these paintings was compromised on the basis of computer analysis of the paintings' fractal dimension [31]. This claim was subsequently disputed by analyzing childlike drawings that supposedly have the same fractal dimension as Pollock paintings [13].

A closely related task is that of painting classification, i.e., the task of assigning a specific artist to a given painting (from a dataset of paintings by two or more artists). Note that authentication can be viewed as a special case of (binary) classification, provided that the dataset consists of paintings by the artist in question. Recent developments in both areas have focused on preprocessing techniques of reducing the high dimensionality of visual data to low-dimensional representations which can be manipulated towards image understanding. This

\footnotetext{
${ }^{1}$ Nathan Netanyahu is also affiliated with the Center for Automation Research, University of Maryland, College Park, MD 20742 (email: nathan@cfar.umd.edu).
}

process is essentially accomplished upon the transition from image space to feature space. The prevailing convention for an effective methodology for this task has been the use of painter-specific low-dimensional features, in conjunction with neural network techniques. Our contribution, on the other hand, attempts at performing image classification using a hybrid genetic algorithm [15]. Specifically, our approach relies on numerous low-dimensional features and uses a weighted $k$ nearest neighbor training procedure to perform the desired classification.

The area of painter classification based on visual data consists of large amounts of data which are the painting images. Within these data lies the answer as to which artist painted a given painting. The task of transforming these data into an answer requires large computing power and good navigation skills through the solution space. Furthermore, the criteria for how connections in the data imply whether a painting was painted by one painter or another is beyond one's grasp. For this kind of problems, the solution is often in the form of a neural network or some other AI method, e.g., a genetic algorithm.

The contribution of an algorithm with a high image classification rate is essential in various applications, such as painting authentication and classification, if only for the financial ramifications of art forgery that could amount to millions of dollars. Moreover, the work presented in this paper could prove applicable to related problems such as handwritten recognition, movement classification, etc. A state where a painter's visual signature is analogous in some sense to a handwritten signature could come sooner than one might expect.

Our initial goal is to achieve a high classification rate (e.g., above $85 \%$ and $75 \%$ for the 2 - and 3-class cases, respectively). We believe this goal could be achieved by adapting a genetic algorithm. The rationale leading to our assumption is that a painting can be represented as an RGB image, which consists of a large quantity of data with various complex "connections" or image features (e.g., statistical descriptors, spatial features, etc.). Since these features are too complex for a human to figure out (as can be deducted from the case of the Vermeer forgeries), it seems that an AI method should be suitable for navigating through the solution space and supply us with either a relevant set of connections (using genetic programming) or the relevance of each selected connection (using a genetic algorithm).

In this paper we present the problem of painter classification and briefly survey recent research that has been conducted in the field. We then explain the feature extraction phase 


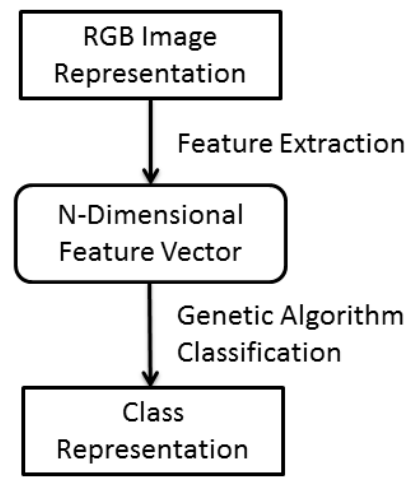

Fig. 1. Typical stages of image classification.

and discuss the various features chosen. Next we present in detail the GA-based classification procedure, and provide empirical results for the 2- and 3-class cases. The results show a significant improvement due to the use of a GA-based scheme (in comparison to a standard nearest-neighbor (NN) classifier).

\section{BACKGROUND}

Image authentication is the task of deciding whether or not a given painting was painted by a specific painter. The related task addressed by us, though, is image classification, i.e., the task of deciding which artist (from a certain group of more than two artists) painted a given painting. The input to our problem consists of painting images of the group of artists (several paintings for each artist), and our objective is to automatically classify a given painting. One of the difficulties in solving this problem is that we cannot define a certain set of rules that the painting has to conform to in order to classify it to the subgroup corresponding to the correct artist. For this reason, computer vision techniques which are capable of identifying shapes and objects in an image are not sufficiently effective for solving the problem.

Each image in our dataset is represented by an RGB matrix of the same resolution in which the painting image was acquired. As a large amount of data is concerned, in order to discover the hidden connections in the images that contain the answer as to whether a painting was painted by one painter or another, we extract a variety of features from the image. This technique is common in the field of content-based classification. Among these features one can find color histograms (RGB, HSV), Fourier spectra, statistical descriptors, Gabor wavelets, fractal dimension, independent components and others. See [32] for details. Subsequent to this process, each painting is represented as a vector whose dimension is the number of features chosen. We later describe our hybrid GA-based NN classification scheme with respect to these features. (See, e.g., [15], [9], [4] for related work on hybrid, GA-based classification.) This process can be viewed as "automatic feature selection" by optimizing feature weights via a GA [29], as will be explained in section V.

\section{A. Past Work}

Formerly there have been attempts to harness the strength of image analysis tools to classify historical art paintings into categories of artists or genres. Keren [16] proposed a framework for image classification, which applies the natural language processing method of a naive-Bayes classifier to local features of an image, by using the coefficients of a discrete cosine transform (DCT). Additionally, Herik and Postma [32] have surveyed several features which are relevant to the historic art domain and concluded that neural-network techniques combined with domain knowledge, were most suitable to the task of automatic image classification. They also argued that "further improvements can be gained by the use of a content-based attentional selection procedure". Kroner et al. [18] applied automatic pattern recognition methods using histograms to classify a drawing to a certain artist. Li and Wang [20] applied a two-dimensional multi-resolution hidden Markov model to classify Chinese ink paintings and form a distinct digital signature. Kammerer et al. [14] presented a strategy for the analysis of under-drawing strokes in infra-red reflectograms, in order to determine the drawing tool used to draft a painting. S. Luo [21] studied a neural-network multi layer perceptron (MLP) with a dataset of X-ray images divided into patches, in order to classify oil paintings. It incorporates implementation of Fourier and wavelet transforms, along with a covariance matrix and statistical momentum as features. (Further relevant details can be found in [22], [6], [17], [1], [3], [23], [5], [7], [10], [12], [31], [24], [19].)

The related work can be divided into authentication problems and classification problems, where the former is a special case of the latter. (Authentication can be viewed as a binary classification of a given painting with respect to an artist in question, i.e., either it was painted by the artist or it was not.) Most of the above surveyed articles focus on a specific type of dataset, ranging from Chinese ink paintings to X-ray images to infra-red reflectograms, and from black and white sketches by Delacroix to impressionistic paintings. Thus the methods described in these articles tend to focus on specific feature selection that is exploited for the classification of a specific type of dataset. In contrast, our approach is not confined to a special case of classification. Nor does it focus on features suitable to a specific domain. Our method enables tossing a large number of features (including very simple ones) at the GA-based classifier, without the need to tailor a unique set of features to the domain space at hand. This is possible due to the strength of the algorithm that constitutes an attentional selection system for image features, based on a natural selection based algorithm.

\section{FEATURES EXTRACTED}

As illustrated in Figure 1, prior to our genetic algorithm there is a preprocessing stage which maps the data into feature vectors. In this section we review several feature types used in our experiments. These features were chosen based on previous painting classification methods. For the purpose of our genetic algorithm, we prefer naturally as many features as possible, as we trust our evolutionary module to attach an appropriate weight to each feature according to its relevance to the problem at hand. In addition to the features discussed below, we used color uniqueness features, defined in terms of unique color 
values in the two color representations. These color uniqueness features represent the variety in pixel values in the different image color representations.

\section{A. Color Histograms}

Color histograms represent the number of pixels in each color value in the span of the representation's color range. For these color spaces we produce 256-dimensional vectors (according to the number of histogram bins). Histograms are extracted separately for 6 dimensions of an image (i.e., red, green, blue, hue, saturation, value) using 256 depth sized bins. Subsequently, double precision feature values are obtained by applying the statistical descriptors described below to the vectors produced by the histograms. Values for the HSV representation are produced from normalized RGB values [30], according to the following code.

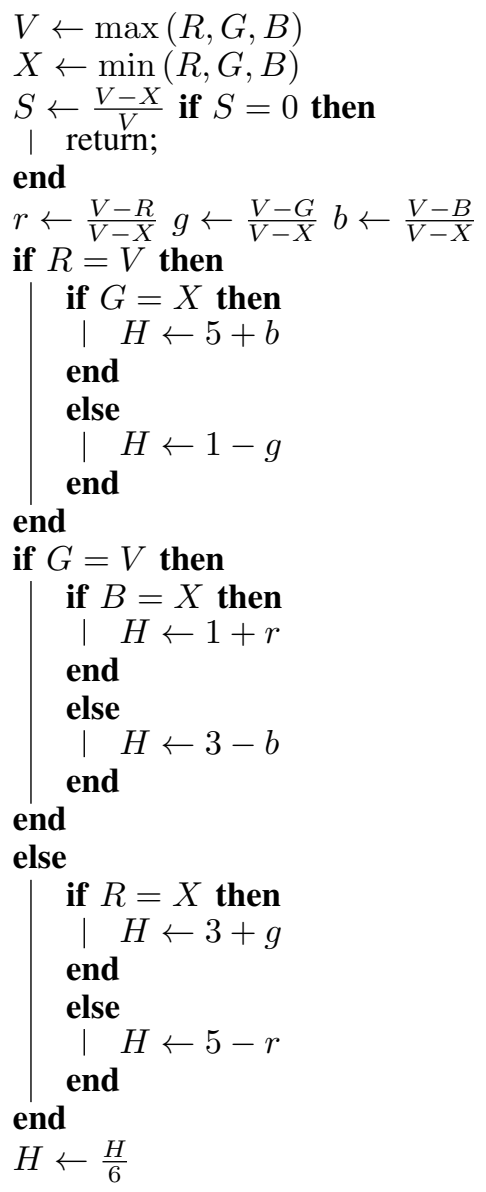

Algorithm 1: Pseudo-code of RGB to HSV mapping.

\section{B. Fourier Spectra}

Fourier's transform is one of the most applied transformations in the field of signal processing. Upon transforming an image matrix, the transform produces a spectral representation of frequencies that form the image. Sine waveforms of amplitudes and phases constitute an image when joined together, and can be inversely transformed to the original image. The transform and its inverse for a vector of length $N$ are computed by:

$$
X(k)=\sum_{j=1}^{N} x(j) \omega_{N}^{(j-1)(k-1)}
$$

$$
x(j)=(1 / N) \sum_{k=1}^{N} X(k) \omega_{N}^{-(j-1)(k-1)}
$$

where $\omega_{N}=e^{(-2 \pi i) / N}$ is an $N$-th root of unity. Amplitudes for the transform are computed by applying an absolute operator on the transformation's matrix.

\section{Fractal Dimension}

Fractal dimension is a feature associated with an image texture. In a nutshell it measures the change of a pattern detail (the fractal) relative to the scale at which it is measured. The concept of fractal dimension is widely used in various areas of applied mathematics (e.g., fractals and chaos theory), biophysics, hydrology, etc. As an approximation to the fractal dimension we calculated an approximate Hurst exponent [27], [28], which is related to the fractal dimension by $D=2-H_{E}$, where $D$ is the fractal dimension and $H_{E}$ is the Hurst exponent.

\section{Steerable Gaussian Filter}

Oriented filters are filters that may be applied and rotated in different angles. Filters of this kind are used in various image processing tasks, such as image enhancement, edge detection and texture analysis. A steerable Gaussian filter evaluates the first directional derivative of an image. It attempts to trace the brush strokes applied by the painter while painting. The filter was implemented using the method outlined in [8] and 24 double precision feature values are extracted from each painting for successive angles separated by $15^{\circ}$.

\section{E. Texture Features}

Texture is an important feature when identifying objects or regions in an image. Data stored in textural features is of the spatial distribution of tonal variations within a band. The texture of an image can be captured by a gray level co-occurrence matrix which provides the distribution of cooccurring values for a given offset. Haralick's texture features (as defined in [11]) are extracted and used in our experiments.

\section{F. Statistical Descriptors}

Statistical descriptors are among the most trivial features that may be extracted in the preprocessing stage. Nonetheless, they may be of high value to the classification of a painting's author. As we trust our algorithm with intelligent weighting of the various preprocessing techniques, we add many trivial statistical descriptors, e.g., standard deviation, skewness, kurtosis, and the minimum, maximum, mean and median values for each of the values of the RGB and HSV representations, and of the amplitudes of the Fourier transform.

Let $I(x, y)$ denote an image value (at a particular pixel) of an $N \times M$ image, for all of the image bands considered (i.e., gray-scale, as well as RGB and HSV bands). We compute the following parameters: 


$$
\begin{gathered}
\text { mean }=\bar{I}=\frac{\sum_{x=1}^{N} \sum_{y=1}^{M} I(x, y)}{N M} \\
\text { stddev }=\sigma=\frac{\sqrt{\sum_{x=1}^{N} \sum_{y=1}^{M}(I(x, y)-\bar{I})^{2}}}{N M-1} \\
\text { skewness }=\frac{\sum_{x=1}^{N} \sum_{y=1}^{M}\left(\frac{I(x, y)-\bar{I}}{\sigma}\right)^{3}}{N M} \\
\text { kurtosis }=\frac{\sum_{x=1}^{N} \sum_{y=1}^{M}\left(\frac{I(x, y)-\bar{I}}{\sigma}\right)^{4}}{N M}-3
\end{gathered}
$$

\section{THE DATASET}

The dataset for our experiments consists of $(3 \times 40=)$ 120 digital reproductions of paintings by Vincent van Gogh, Rembrandt van Rijn and Pierre Auguste Renoir, downloaded from the Webmuseum [26]. The images are 24-bit color depth with varying resolutions averaged approximately at $1000 \times$ 1000 pixels, and compressed as JPEG formatted files. Amongst the images one can find various sceneries and portraits with a diverse variety of colors and styles. Titles of the paintings in the dataset used in our experiments appear in the Appendix.

It is important to maintain some uniformity, in terms of image acquisition, e.g., to ensure that the same camera is used to acquire all painting images. Otherwise, the classification algorithm might classify the electronic device used to acquire a painting, rather than the painter itself. Additionally, image digitization introduces various artifacts and distortions, resulting in unavoidable noise that is an integral part of any reproduction process. These issues should not pose a problem as long as they are consistent throughout the dataset. Indeed, this is the case of our dataset which consists of images that were taken by the same photographer, using the same digitization process. On top of that, compression sourced artifacts may appear due to the use of a lossy JPEG compression. This does not pose a problem as the compression factor is fixed across the images and the quality is high enough so that the artifacts are negligible.

\section{PROPOSED APPROACH}

We now explain our mapping of the painter classification problem to a GA framework. As sketched before, each painting image in the dataset of $(3 \times 40=) 120$ images is transformed into a 78-dimensional feature vector, according to the number of features extracted. (Recall, we use 78 double precision feature values, according to the discussion in section III.) The features consist of $(5 \times 6=) 30$ values of the mean, median, maximum, minimum and the number of histogram bins with non-zero frequency of each band of the RGB and HSV color representations. In addition, we take into account $(4 \times 2)=8$ values of the standard deviation, skewness, kurtosis and mean of the gray-scale and FFT images, as well as the number

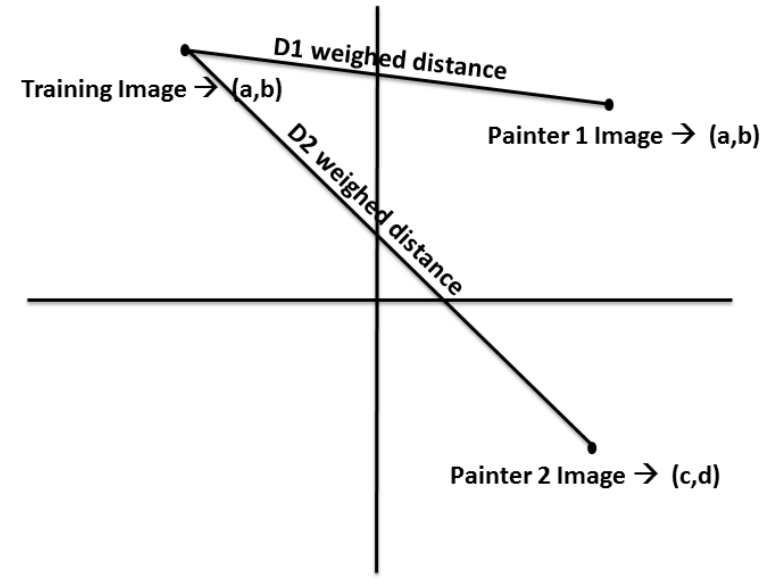

Fig. 2. Simplified two-dimensional feature space, illustrating the notion of weighted distance between a (point representing a) training image and two points representing images by two painters.

of unique RGB color triplets, and the Hurst exponent, 24bin histogram of directional derivatives, and Haralick's texture features (in the gray-scale image).

We need to consider an appropriate representation that could be used by a genetic algorithm. In order to do so, we define a chromosome as follows. To distinguish ultimately between different painters, we will attempt to attach relevant weights to the features used. (We show later how these weights are used in a weighted nearest neighbor procedure for fitness evaluation.) Thus, a natural representation of a solution would be a chromosome which consists of 78 double precision values. As mentioned, we shall apply these weights in the validation stage of the learning procedure and use the weights of the best chromosome obtained to evaluate the performance of our genetic algorithm. The basic framework is as follows:

1) Initialize vectors to be used with the nearest neighbor algorithm.

2) Train a genetic algorithm using a fitness function based on the previously produced vectors and the weighted nearest neighbor algorithm.

3) Validate performance of the best chromosome spawned by the genetic algorithm by comparing its performance to that of an ordinary (unweighted) nearest neighbor algorithm.

Our dataset is divided to $(30 \times 3)=90$ training images and $(10 \times 3)=30$ validation images. Additionally, the image subset for training was divided to $(5 \times 3)=15$ paintings used for NN classification (as explained in the next subsection), and $(25 \times 3)=75$ paintings used to train the GA. We have experimented with two classification scenarios: 3-way classification of van Gogh, Rembrandt and Renoir, and 2-way classifications of their three permutations. We elaborate below on each of the above stages.

\section{A. Nearest Neighbor Initialization}

A portion of the training data is used for an initialization of vectors that are used by the nearest neighbor algorithm. A simple two-dimensional example is depicted in Figure 2. 
Suppose that the vectors, "Painter 1 Image" and "Painter 2 Image", are added to the feature space and are subsequently utilized in the fitness evaluation of the chromosomes and in the verification stage. During this process, weighted distances with respect to a given chromosome are calculated between the initialized vectors and image vectors used to train the GA.

The nearest neighbor algorithm is a special case of the general $k$-nearest neighbor algorithm (where $k=1$ ). It is a method for classifying objects based on the closest training examples in feature space. The training stage consists of storing labeled training vectors, and the classification is accomplished by calculating which vector label is the most frequent among the $k$ closest vectors to a feature vector in question. Based on our experiments, we choose $k$ as 1 , using Euclidean distance as a distance metric.

\section{B. Training of Genetic Algorithm}

The training phase of the genetic algorithm employs the representation of the problem as a chromosome, and the definition of several evolutionary operators that are used during the genetic simulation performed by the genetic algorithm. These operators include crossover and mutation (see below). Also, the evolution is customized by fine-tuning parameters and flags such as crossover/mutation rates and elitism.

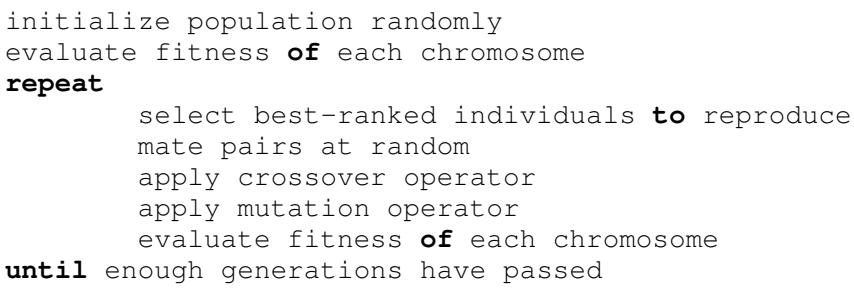

Fig. 3. Pseudo-code of genetic algorithm.

As illustrated before, our chromosome represents double precision values that are vector components in feature space. Each chromosome defines a solution by employing its feature weights for calculating the distances between a newly inserted vector (extracted from a painting in question) to all the feature vectors in the nearest neighbor dataset, according to Eq. (7). The weighted distance is dependent on the chromosomes' parameters as weights, and is defined by:

$$
N N_{w}=\sqrt{\sum_{i=1}^{d} w_{i}\left(y_{i}-z_{i}\right)^{2}}
$$

where $Y=\left(y_{1}, y_{2}, \ldots, y_{d}\right)$ and $Z=\left(z_{1}, z_{2}, \ldots z_{d}\right)$ are painting representations in feature space, and $w_{i}(i=1, \ldots, d)$ are the weights corresponding to a given chromosome. Figure 4 illustrates a chromosome structure, which consists of the various weights used by the nearest neighbor classifier.

Let us now define the fitness function to be used during the training phase of the genetic algorithm. The procedure in Figure 5 evaluates the fitness of a chromosome as the number of correct painting classifications obtained by weighted distance computations between every feature vector and all

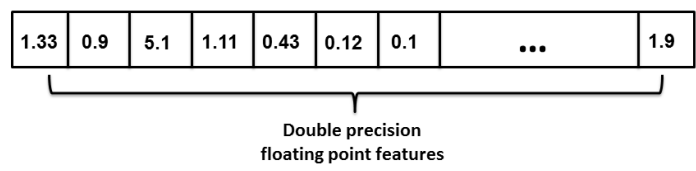

Fig. 4. An example of a chromosome containing 78 feature values. Every cell contains the coefficient of its appropriate feature, and the 78-value chromosome is a solution for the classification weights applied by the weighted nearest neighbor method.

other vectors in the "nearest neighbor" subset. This procedure is called every time a chromosome's fitness is evaluated.

Additionally, the crossover operator is based on a doublepoint location, i.e., two locations in the chromosome are chosen randomly and the data passed to their offspring changes its source at each randomly chosen location. One offspring would consist of "father-mother-father" configuration and the other of the complementary structure "mother-father-mother". The mutation operator used is an additive random perturbation (of some Gaussian distribution) to each chromosome gene (i.e., a double precision number generated at random).

We also set certain probabilities to our GA. Specifically, we set the probability of crossover occurrence to $90 \%$, i.e., in selecting mating chromosomes for offspring production of the next generation, $10 \%$ of these offspring would be identical to their parents. Mutation rate is set to $35 \%$, which is relatively high compared to its common value in other genetically solved problems, but was found effective empirically. Additionally, we set the population size in each generation to 250 chromosomes. We also applied elitism, which captures the notion of passing - as is - the two best chromosomes of each generation to the next.

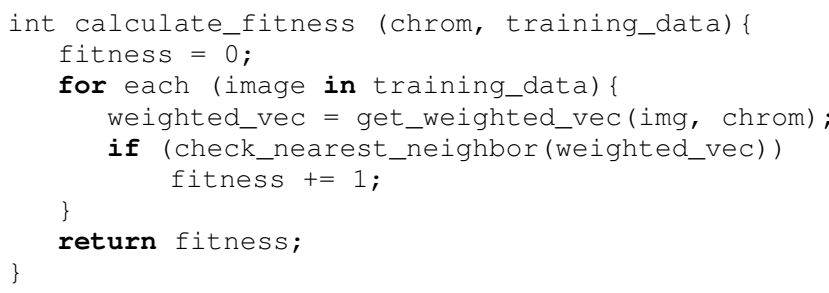

Fig. 5. Pseudo-code of fitness evaluation during training.

\section{Performance Validation}

Subsequent to the training phase of our genetic algorithm, validation is performed on $(10 \times 3)=30$ images of the dataset by applying the best chromosome obtained, i.e., the one with the highest fitness value after 50 generations. Note that these images were not involved in any of the previous phases of the experiment, thus ensuring they were not used for training of the genetic algorithm. Each vector (chosen for validation) is compared against all of the vectors in the dataset (stored during the initialization phase), by applying the weights of the best chromosome to find the vector's (weighted) nearest neighbor. The vector is classified according to its (weighted) nearest neighbor label. We can thus classify all of the "validation 
vectors" and evaluate the performance according to the number of correctly classified vectors.

Aside from evaluating our genetic algorithm-based classification, we compare its performance to that of a standard (unweighted) nearest neighbor classifier. See next section for specific results.

\section{EMPIRICAL RESULTS}

We have implemented our scheme using [2] as a platform for the genetic algorithm and MATLAB as an infrastructure for calculating feature values. Specifically, we calculate 78 feature values as described in section III. The dataset experimented with consists of $(40 \times 3)=120$ images of paintings by Rembrandt, van Gogh, and Renoir. We invoked 10 random runs, where each run was restarted with random initial chromosomes. It would be of interest, of course, to test empirically the impact of the number of runs on the algorithm's overall performance. The best chromosome over these 10 runs was chosen for a given subset of the training images. Additionally, cross-validation was conducted by shuffling (at random) the role of the images, i.e., images previously used for training were used for validation in a subsequent run and vice versa. This way we utilized effectively our dataset. Averaging the cross-validation results, we obtained a classification accuracy of roughly $90 \%$ and $80 \%$ for the 2-painter case and the 3painter case, respectively. These preliminary results exceed by far the results we have obtained for the plain, unweighted nearest neighbor version ( $\sim 70 \%$ and $\sim 65 \%$, respectively, for the 2painter and the 3-painter cases). The results for the unweighted nearest neighbor version were obtained concurrently to the experiments with the weighted nearest neighbor algorithm. They were acquired using the same image subsets (for training and validation). Thus we can observe the eminent improvement achieved using the weighted nearest neighbor method, trained by our genetic algorithm. Examining the algorithm's output chromosomes reveals that every feature is important when attempting to classify the painter in question, as very small weights were rarely encountered in practice. The graphs in Figure 6 present the fitness function associated with the best and average chromosomes in a single run over 50 generations. The evolutionary improvement is clearly evident.

\section{CONCLUSION}

The problem of automatic painter classification has gained much attention over the past decades. Much progress has been made with regards to both relevant preprocessing techniques and classification algorithms adopted from various fields such as natural language processing and pattern recognition. Still, the problem of painter classification remains a complex task that requires more sophisticated techniques. Improvement in classification procedures may aid many practical applications other than the presented painter classification problem. These applications include medical imaging analysis, gene expression of micro-array data and signature recognition.

In this paper we have discussed the usage of a genetic algorithm that utilizes the weighted NN algorithm in order to classify historic paintings. The technique presented shows promising preliminary results and may prove useful in various types of datasets other than historic paintings. We surveyed

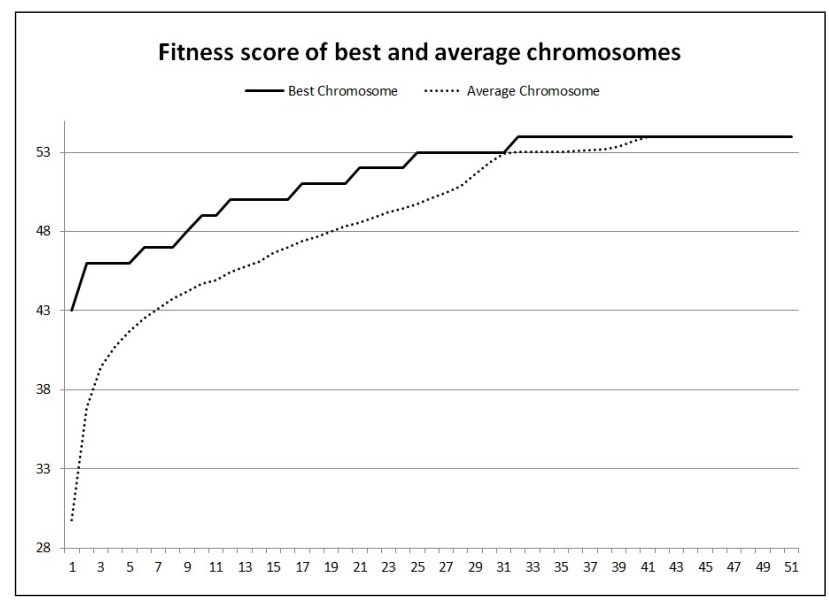

Fig. 6. Fitness scores of best and average chromosomes over 50 generations: The vertical axis represents the fitness score in each generation and the horizontal axis represents the generation number; a chromosome's fitness is defined as the number of images classified correctly using that chromosome's weights. Fitness scores range from 0 to $(25 \times 3)=75$. In the 3 -class problem, the fitness is incremented by 1 for each correct classification (by the weighted nearest neighbor procedure) against a training subset of 15 painting images (per painter)

related work that employs various techniques, each of which tends to focus on a specific dataset type. Moreover, we presented preprocessing methods that we have used in our classification algorithm, and a mapping of the problem that can be solved by our genetic scheme. The problem mapping, of course, is an essential component in the design of a genetic algorithm that exploits effectively the evolutionary process. Finally, we described a hybrid genetic framework for achieving an efficient classification procedure.

The use of a genetic algorithm for learning feature effectiveness via a generic weighted procedure holds within itself a potential improvement of classification performance in all image classification tasks. This kind of attentional selection procedure has the power to perceive classifying image details in places where the human mind is not capable of looking. It is possible also that coming up with enhanced preprocessing techniques can be done effectively by genetic programming or a similar paradigm harnessing the enormous computing power of current machines.

\section{REFERENCES}

[1] J.B. Adams, D.E. Sabol, V. Kapos, R.A. Filho, D.A. Roberts, M.O. Smith, and A.R. Gillespie. Classification of multispectral images based on fractions of endmembers: Application to land-cover change in the Brazilian Amazon. Remote Sensing of Environment, 52(2):137-154, 1995.

[2] S. Adcock. Gaul - Genetic Algorithms Utility Library. http://gaul. sourceforge.net, 2000.

[3] R. Barandela and M. Juarez. Supervised classification of remotely sensed data with ongoing learning capability. International Journal of Remote Sensing, 23(22):4965-4970, 2002.

[4] Y. Bengio, Y. LeCun, C. Nohl, and C. Burges. LeRec: A NN/HMM hybrid for on-line handwriting recognition. Neural Computation, 7(6):1289-1303, 1995.

[5] D. Chen and D. Stow. The effect of training strategies on supervised classification at different spatial resolutions. Photogrammetric Engineering and Remote Sensing, 68(11):1155-1162, 2002. 
[6] F.J. Cortijo and N.P. De La Blanca. A comparative study of some non-parametric spectral classifiers. applications to problems with highoverlapping training sets. International Journal of Remote Sensing, 18(6):1259-1275, 1997.

[7] Q. Du and C.I. Chang. A linear constrained distance-based discriminant analysis for hyperspectral image classification. Pattern Recognition, 34(2):361-373, 2001.

[8] W.T. Freeman and E.H. Adelson. The design and use of steerable filters. IEEE Transactions on Pattern Analysis and Machine Intelligence, 13(9):891-906, 1991.

[9] D.E. Goldberg and J.H. Holland. Genetic algorithms and machine learning. Machine Learning, 3(2):95-99, 1988.

[10] R. Haapanen, A.R. Ek, M.E. Bauer, and A.O. Finley. Delineation of forest/nonforest land use classes using nearest neighbor methods. Remote Sensing of Environment, 89(3):265-271, 2004.

[11] R.M. Haralick, K. Shanmugam, and I.H. Dinstein. Textural features for image classification. IEEE Transactions on Systems, Man and Cybernetics, (6):610-621, 1973.

[12] C.W. Hsu and C.J. Lin. A comparison of methods for multiclass support vector machines. IEEE Transactions on Neural Networks, 13(2):415425, 2002.

[13] K. Jones-Smith and H. Mathur. Fractal analysis: revisiting Pollock's drip paintings. Nature, 444(7119):E9-E10, 2006.

[14] P. Kammerer, M. Lettner, E. Zolda, and R. Sablatnig. Identification of drawing tools by classification of textural and boundary features of strokes. Pattern Recognition Letters, 28(6):710-718, 2007.

[15] J.D. Kelly and L. Davis. A hybrid genetic algorithm for classification. In Proceedings of the 12th International Joint Conference on Artificial Intelligence, pages 645-650, 1991.

[16] D. Keren. Painter identification using local features and naive Bayes. In Proceedings of the IEEE International Conference on Pattern Recognition, volume 2, pages 474-477, 2002.

[17] J.L. Kirsch and R.A. Kirsch. The anatomy of painting style: Description with computer rules. Leonardo, pages 437-444, 1988.

[18] S. Kroner and A. Lattner. Authentication of free hand drawings by pattern recognition methods. In Proceedings of the IEEE 14th International Conference on Pattern Recognition, volume 1, pages 462 464, 1998.

[19] B. Lam and V. Ciesielski. Discovery of human-competitive image texture feature extraction programs using genetic programming. In Proceedings of the Genetic and Evolutionary Computation Conference, pages 1114-1125, 2004.

[20] J. Li and J.Z. Wang. Studying digital imagery of ancient paintings by mixtures of stochastic models. IEEE Transactions on Image Processing, 13(3):340-353, 2004.

[21] S. Luo. Oil painting classification. http://homepages.cae.wisc.edu/ $\sim$ ece539/fall10/project/Luo_pro.pdf, 2010

[22] S. Lyu, D. Rockmore, and H. Farid. A digital technique for art authentication. Proceedings of the National Academy of Sciences of the United States of America, 101(49):17006-17010, 2004.

[23] G. Mercier and M. Lennon. Support vector machines for hyperspectral image classification with spectral-based kernels. In Proceedings of the International IEEE Geoscience and Remote Sensing Symposium, volume 1, pages 288-290, 2003.

[24] R. Paredes and E. Vidal. Learning weighted metrics to minimize nearest-neighbor classification error. IEEE Transactions on Pattern Analysis and Machine Intelligence, 28(7):1100-1110, 2006.

[25] D. Phillips. How do forgers deceive art critics? The Artful Eye. R. Gregory, J. Harris, P. Heard, and D. Rose, Eds., Oxford University Press, pages 372-388, 1995.

[26] N. Pioch. Webmuseum. http://webmuseum.meulie.net/wm/, 1994.

[27] J.C. Russ. Surface characterization: Fractal dimensions, Hurst coefficients, and frequency transforms. Journal of Computer-Assisted Microscopy, 2(3):161-183, 1990.

[28] B. Schiele and J. Crowley. Object recognition using multidimensional receptive field histograms. In Proceedings of the European Conference on Computer Vision, pages 610-619, 1996.
[29] W. Siedlecki and J. Sklansky. A note on genetic algorithms for largescale feature selection. Pattern Recognition Letters, 10(5):335-347, 1989.

[30] A.R. Smith. Color gamut transform pairs. In ACM Siggraph Computer Graphics, volume 12, pages 12-19, 1978.

[31] R.P. Taylor, R. Guzman, T.P. Martin, G.D.R. Hall, A.P. Micolich, D. Jonas, B.C. Scannell, M.S. Fairbanks, and C.A. Marlow. Authenticating Pollock paintings using fractal geometry. Pattern Recognition Letters, 28(6):695-702, 2007.

[32] H.J. van den Herik and E.O. Postma. Discovering the visual signature of painters. Future Directions for Intelligent Systems and Information Sciences. N. Kasabov, Ed., Physica-Verlag, Heidelberg, pages 129-147, 2000 . 


\section{APPENDIX}

This appendix lists the $(40 \times 3)=120$ titles of the paintings experimented with by van Gogh, Rembrandt, and Renoir.

\begin{tabular}{|c|c|c|c|}
\hline$\#$ & van Gogh & Rembrandt & Renoir \\
\hline 1 & bandaged-ear & abraham & apres-bain \\
\hline 2 & berceuse & anslo & baigneuses \\
\hline 3 & cordeville & aristotle-homer & bathers-1887 \\
\hline 4 & corridor-asylum & artemis & bathers-1918 \\
\hline 5 & cypress-star & bathing-river & bougival \\
\hline 6 & cypresses & bathsheba & canoeist \\
\hline 7 & flower-beds-holland & belshazzar & chocquet \\
\hline 8 & green-vineyard & children & city \\
\hline 9 & green-wheat-field & danae & country \\
\hline 10 & house-ploughman & david & dancer \\
\hline 11 & mme-trabuc & descent & durieux \\
\hline 12 & mr-trabuc & emmaus & flowers \\
\hline 13 & old-mill & hendrickje & gabrielle \\
\hline 14 & old-vineyard & holy-family & girl-seated \\
\hline 15 & olive-alpilles & jan-six & jugglers \\
\hline 16 & olive-trees & magn-glass & lady-piano \\
\hline $17 \mathrm{o}$ & orchard-bloom-poplars & meditation & laundress \\
\hline 18 & orchard-plum-trees & mill & loge \\
\hline 19 & poppies & music-party & lucie-berard \\
\hline 20 & red-vineyard & nicolaes-tulp & near-lake \\
\hline 21 & reminiscences & old-man & fournaise \\
\hline 22 & road-menders & ostrich & horsewoman \\
\hline 23 & roulin & potiphar & meadow \\
\hline 24 & self-1 & prodigal-son & moulin-galette \\
\hline 25 & self-2 & raising-lazarus & nini \\
\hline 26 & self-easel & .1640 & parapluies \\
\hline 27 & self-gauguin & .1661 & premiere-sortie \\
\hline 28 & self-orsay & .1669 & promenade \\
\hline 29 & self-whitney & .night-watch & ride \\
\hline 30 & skull-cigarette & return-prodigal-son & romain-lacaux \\
\hline 31 & sun-cloud & ruts & sisley-wife \\
\hline 32 & threatening-skies & samson & women \\
\hline 33 & trees-asylum & scholar & seashore \\
\hline 34 & trees-ivy-asylum & self-1629 & seated-bather \\
\hline 35 & village-stairs & self-1634 & sewing \\
\hline 36 & wheat-field & self-1660 & sisley \\
\hline 37 & wheat-rising-sun & slaughtered-ox & swing \\
\hline 38 & willows & staalmeesters & terrace \\
\hline 39 & peasant & stofells & watercan \\
\hline 40 & woman-arles & tobias & woman-veil \\
\hline
\end{tabular}

